\title{
CANONICAL MAPS OF SURFACES DEFINED BY ABELIAN COVERS*
}

\author{
RONG $\mathrm{DU}^{\dagger}$ AND YUN GAO
}

\begin{abstract}
In this paper, we classified the surfaces whose canonical maps are abelian covers over $\mathbb{P}^{2}$. Moveover, we give defining equations for Perssson's surface and Tan's surfaces with odd canonical degrees explicitly.
\end{abstract}

Key words. Canonical map, abelian cover.

AMS subject classifications. 14J29, 14E20.

1. Introduction. Let $X$ be a minimal surface of general type over $\mathbb{C}$. As usual, let $p_{g}, q, \chi\left(\mathcal{O}_{X}\right), K_{X}^{2}$ be the numerical invariants of $X$,

$$
\varphi_{K_{X}}: X \rightarrow \Sigma \subset \mathbb{P}^{p_{g}-1}
$$

be the canonical map of $X$ and $d=\operatorname{deg} \varphi_{K_{X}}$ be the canonical degree of $X$. It is well known that the behavior of $\varphi_{K_{X}}$ is quite complicated, however the degree of it can not be too large. In 1979, Beauville ([Bea]) proved that when $\varphi_{K_{X}}$ is a generically finite map, the degree is at most 36. Furthermore, $\operatorname{deg} \varphi_{K_{X}}=36$ if and only if $K_{X}^{2}=36$, $p_{g}=3, q=0, \Sigma=\mathbb{P}^{2}$ and $\left|K_{X}\right|$ is base point free. Later, Xiao also found some restrictions on surfaces with high canonical degrees ([Xiao]). For surfaces of general type, since the canonical degree is bounded above, it is interesting to know which positive integers $d$ 's occur as the degree of the canonical map. Among all known surfaces with highest canonical degree less than 36 is the surface with canonical degree 16 which was constructed by Persson([Per]) as follows. let $\pi: X \rightarrow Y$ be a double cover of a Campedelli surface $Y$ branched along $2 K_{Y}$, where the Campedelli surface is the surface of general type with $p_{g}=q=0, K_{Y}^{2}=2$, and $\left|2 K_{Y}\right|$ is base point free. He found that $\varphi_{K_{X}}=\pi \circ \varphi_{K_{Y}}$, hence $\varphi_{K_{X}}$ is degree 16 over $\mathbb{P}^{2}$. We see that his construction is based on the the existence of Campedelli surface, however the construction of a Campedelli surface is not an easy work ([Pet]). The remaining problem that whether there exists a surface of general type over $\mathbb{P}^{2}$ with canonical degree of the gap between 17 and 35 is still open.

If $\Sigma$ is a canonical surface, there are plenty of examples (see [Bea], [Cat1], [V-Z] ) with canonical degrees being 2. For $d=3$ and $d=5$, Tan ([Tan]) and Pardini ([Par1]) constructed several surfaces independently. When $p_{g}(\Sigma)=0$, Beauville ([Bea]) constructed surfaces with $\chi\left(\mathcal{O}_{X}\right)$ arbitrarily large and the canonical degrees 2, 4, 6 and 8 . For $d=9$, Tan also constructed a surface in [Tan]. However, as far as we know that it is still not clear which positive integers $d$ 's occur as the degree of the canonical map even when $\Sigma=\mathbb{P}^{2}$.

\footnotetext{
*Received September 22, 2012; accepted for publication November 9, 2012.

$\dagger$ Department of Mathematics, Shanghai Key Laboratory of PMMP, East China Normal University, Shanghai 200241, P. R. China (rdu@math.ecnu.edu.cn). Current address: Department of Mathematics, The University of Hong Kong, Pokfulam, Hong Kong (rongdu@hku.hk). The research sponsored by The National Natural Science Foundation of China, Shanghai Pujiang Program and the Scientific Research Foundation for the Returned Overseas Chinese Scholars, State Education Ministry.

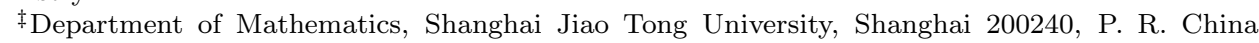
(gaoyunmath@sjtu.edu.cn). The research sponsored by National Natural Science Foundation of China and The Innovation Program of Shanghai Municipal Education Commission.
} 
In this paper, we show that if the canonical map is an abelian cover over $\mathbb{P}^{2}$ then the only possible canonical degrees of a surface of general type are 2, 3, 4, 6, 8, 9, 16 by explicit constructions. Moreover, by using abelian cover, we list the defining equations for Perssson's surface and Tan's surfaces with odd canonical degrees.

We list our main theorems as follows.

Theorem 1.1. Assume $\varphi: X \longrightarrow \mathbb{P}^{2}$ is an abelian cover.

(1) Let $\operatorname{deg} \varphi=16$, then $\varphi=\varphi_{K_{X}}$ if and only if $X$ is defined by

$$
z_{1}^{2}=\ell_{1} \ell_{3} \ell_{4} \ell_{7}, \quad z_{2}^{2}=\ell_{1} \ell_{2} \ell_{4} \ell_{5}, \quad z_{3}^{2}=\ell_{1} \ell_{2} \ell_{3} \ell_{6}, \quad z_{4}^{2}=\ell_{2} \ell_{5} \ell_{6} \ell_{8} .
$$

In particular, the surface defined by the first three equations is a Campedelli surface.

(2) Let $\operatorname{deg} \varphi=9$, then $\varphi=\varphi_{K_{X}}$ if and only if $X$ is defined by $z_{1}^{3}=a_{1} a_{2}^{2}, z_{2}^{3}=$ $a_{1} a_{2} a_{3}$.

(3) Let $\operatorname{deg} \varphi=8$, then $\varphi=\varphi_{K_{X}}$ if and only if $X$ is defined by either $z_{1}^{2}=$ $\ell_{1} \ell_{2} \ell_{7} \ell_{8}, z_{2}^{2}=\ell_{3} \ell_{4} \ell_{7} \ell_{8}, z_{3}^{2}=\ell_{5} \ell_{6} \ell_{7} \ell_{8}$, or $z_{1}^{2}=a_{1} a_{4}, z_{2}^{2}=a_{2} a_{4}, z_{3}^{2}=a_{3} a_{4}$, or $z_{1}^{2}=a_{1} a_{2}, z_{2}^{4}=a_{2}^{3} a_{3}$.

(4) Let $\operatorname{deg} \varphi=6$, then $\varphi=\varphi_{K_{X}}$ if and only if $X$ is defined by $z_{1}^{2}=a_{1} a_{2}, z_{2}^{3}=$ $a_{2} a_{3}^{2}$.

(5) Let $\operatorname{deg} \varphi=4$, then $\varphi=\varphi_{K_{X}}$ if and only if $X$ is defined by either $z^{4}=a_{1}^{2} b_{2}^{3}$,or $z_{1}^{2}=b_{1}, z_{2}^{2}=b_{2}$.

(6) Let $\operatorname{deg} \varphi=3$, then $\varphi=\varphi_{K_{X}}$ if and only if $X$ is defined by either $z^{3}=c_{1}^{2}$,or $z^{3}=c_{2}$.

(7) Let $\operatorname{deg} \varphi=2$, then $\varphi=\varphi_{K_{X}}$ if and only if $X$ is $z^{2}=h$.

Here $\ell_{i}$ 's define different lines and there are at most three lines among them passing through one point, for $i=1 \cdots 8 ; a_{i}$ 's are reduced of degree $2 ; b_{i}$ 's are reduced of degree $4 ; c_{1}, c_{2}$ are reduced of degree $6 ; h$ is reduced of degree 8 and all the irreducible components of $a_{i}$ 's,$b_{i}$ 's, $c_{1}, c_{2}$ and $h$ are simply normal crossing.

THEOREM 1.2. If

$$
\varphi=\varphi_{K_{X}}: X \longrightarrow \mathbb{P}^{2}
$$

is an abelian cover, then $d$ is equal to $2,3,4,6,8,9$, or 16 . In particular, if the canonical degree of $X$ is 36 , then $\varphi_{K_{X}}$ can not be an abelian cover.

In section 2. we present the main facts on abelian covers which are the key points for solving our problem. In section 3, we prove our main theorems. The defining equations of Tan's examples will be given in section 4 .

The authors would like to thank Professor S.-L. Tan for valuable discussions and noticing us his examples([Tan]). We are also grateful to Professor R. Pardini for having told us some relevant references and their paper about classifying Compedelli surfaces with fundamental group of order 8 ([ML-P-R $]$ ).

2. Abelain covers. The theory of cyclic covers of algebraic surfaces was studied first by Comessatti in [Com]. Then F. Catanese ([Cat2]) studied smooth abelian covers in the case $\left(\mathbb{Z}_{2}\right)^{\oplus 2}$ and R. Pardini ([Par2]) analyzed the general case. In this section, we shall recall some basic definitions and results for abelian covers which will facilitate our subsequent discussion. Since our point of view is to find the defining equations for covering surfaces by explicit calculation, we use the expressions and notations appearing in [Gao]. 
Let $\varphi: X \rightarrow Y$ is an abelian cover associated to abelian group $G \cong \mathbb{Z}_{n_{1}} \oplus \cdots \oplus \mathbb{Z}_{n_{k}}$, i.e., function field $\mathbb{C}(X)$ of $X$ is an abelian extension of the rational function field $\mathbb{C}(Y)$ with Galois group $G$. Without lose of generality, we can assume $n_{1}\left|n_{2} \cdots\right| n_{k}$.

Definition 2.1. The dates of abelian cover over $Y$ with group $G$ are $k$ effective divisors $D_{1}, \cdots, D_{k}$ and $k$ linear equivalent relations

$$
D_{1} \sim n_{1} L_{1}, \cdots, D_{k} \sim n_{k} L_{k} .
$$

Let $\mathscr{L}_{i}=\mathscr{O}_{Y}\left(L_{i}\right)$ and $f_{i}$ be the defining equation of $D_{i}$, i.e., $D_{i}=\operatorname{div}\left(f_{i}\right)$, where $f_{i} \in H^{0}\left(Y, \mathscr{L}_{i}^{n_{i}}\right)$. Denote $\mathbf{V}\left(\mathscr{L}_{i}\right)=\operatorname{Spec} S\left(\mathscr{L}_{i}\right)$ to be the line bundle corresponding to $\mathscr{L}_{i}$, where $S\left(\mathscr{L}_{i}\right)$ is the sheaf of symmetric $\mathscr{O}_{Y}$ algebra. Let $z_{i}$ be the fiber coordinate of $\mathbf{V}\left(\mathscr{L}_{i}\right)$. Then the abelian cover can be realized by the normalizing of surface $V$ defined by the system of equations

$$
z_{1}^{n_{1}}=f_{1}, \cdots, z_{k}^{n_{k}}=f_{k} .
$$

So we have the following diagram:

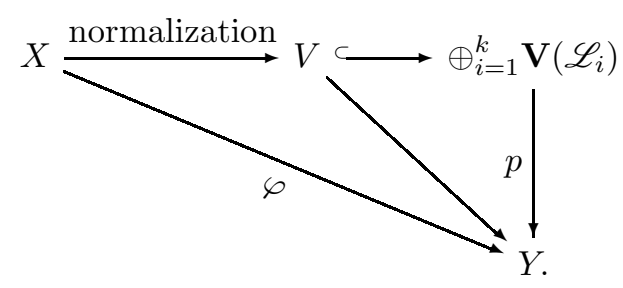

Sometimes we call $X$ is defined by these equations if there is no confusions in the context.

We summerize our main results as follows.

Theorem 2.2. (See [Gao]) Denote by $[Z]$ the integral part of a $\mathbb{Q}$-divisor $Z$, $L_{g}=-\sum_{i=1}^{k} g_{i} L_{i}+\left[\sum_{i=1}^{k} \frac{g_{i}}{n_{i}} D_{i}\right]$.Then

$$
\varphi_{*} \mathcal{O}_{X}=\bigoplus_{g \in G} \mathcal{O}_{Y}\left(-L_{g}\right)
$$

where $g=\left(g_{1}, \cdots, g_{k}\right) \in G$.

So the decomposition of $\varphi_{*} \mathcal{O}_{X}$ is totally determined by the abelian cover.

By Theorem 2.2 we can get following corollary.

Corollary 2.3. If $X$ is non-singular, $D$ is the divisor on $Y$, then

$$
h^{i}\left(X, \varphi^{*} \mathcal{O}_{Y}(D)\right)=\sum_{g \in G} h^{i}\left(Y, \mathcal{O}_{Y}\left(D-L_{g}\right)\right)
$$

The following result will be used to calculate the ramification index.

TheOREm 2.4. Let $P$ be an irreducible and reduced hypersurface in $Y$, let $\bar{P}=$ $\pi^{-1}(P)$ be the reduced preimage of $P$ in $X$, and let $a_{i}$ be the multiplicity of $P$ in $D_{i}=\operatorname{div}\left(f_{i}\right)$. Then

$$
\pi^{*} P=\frac{|G|}{d_{P}} \bar{P}
$$


where

$$
d_{P}=\operatorname{gcd}\left(|G|,|G| \frac{a_{1}}{n_{1}}, \cdots,|G| \frac{a_{k}}{n_{k}}\right)
$$

is the number of points in the preimage of a generic point on $P$.

3. Canonical map. Let $X$ be a surface of general type whose canonical map $\varphi_{K_{X}}$ is a generically finite cover of a surface in $\mathbb{P}^{2}$.

Lemma 3.1. Assume $\varphi: X \rightarrow \mathbb{P}^{2}$ is a finite cover of degree $36, \varphi_{K_{X}}=\varphi$ if and only if

$$
\varphi_{*} \mathcal{O}_{X}=\mathcal{O}_{\mathbb{P}^{2}} \oplus E \oplus \mathcal{O}_{\mathbb{P}^{2}}(-4),
$$

where $E$ is a rank 34 bundle satisfying $E^{\vee} \cong E(4)$ and $H^{0}(E(1))=0$.

Proof. If $\varphi$ is a finite cover, we take $E_{0}$ as the trace free part of $\varphi_{*} \mathcal{O}_{X}$, so $\varphi_{*} \mathcal{O}_{X}=\mathcal{O}_{\mathbb{P}^{2}} \oplus E_{0}$. By relative duality,

$$
\varphi_{*} \omega_{X} \cong\left(\varphi_{*} \mathcal{O}_{X}\right)^{\vee} \otimes \omega_{\mathbb{P}^{2}}=\mathcal{O}_{\mathbb{P}^{2}}(-3) \oplus E_{0}^{\vee}(-3) .
$$

Since $\omega_{X}=\varphi^{*}\left(\mathcal{O}_{\mathbb{P}^{2}}(1)\right)$, by projection formula, $\varphi_{*} \omega_{X}=\varphi_{*} \varphi^{*}\left(\mathcal{O}_{\mathbb{P}^{2}}(1)\right)=\mathcal{O}_{\mathbb{P}^{2}}(1) \otimes$ $\varphi_{*} \mathcal{O}_{X}$, so

$$
\mathcal{O}_{\mathbb{P}^{2}}(1) \oplus E_{0}(1) \cong \mathcal{O}_{\mathbb{P}^{2}}(-3) \oplus E_{0}^{\vee}(-3)
$$

We see that $E_{0}=E \oplus \mathcal{O}_{\mathbb{P}^{2}}(-4)$, and $E^{\vee}=E(4)$.

On the other hand, if $\varphi_{*} \mathcal{O}_{X}=\mathcal{O}_{\mathbb{P}^{2}} \oplus E \oplus \mathcal{O}_{\mathbb{P}^{2}}(-4)$, then

$$
\begin{aligned}
\varphi_{*}\left(\omega_{X} \otimes \varphi^{*} \mathcal{O}_{\mathbb{P}^{2}}(-1)\right) & =\varphi_{*} \omega_{X} \otimes \mathcal{O}_{\mathbb{P}^{2}}(-1) \\
& =\left(\varphi_{*} \mathcal{O}_{X}\right)^{\vee} \otimes \omega_{\mathbb{P}^{2}} \otimes \mathcal{O}_{\mathbb{P}^{2}}(-1) \\
& =\left(\mathcal{O}_{\mathbb{P}^{2}} \oplus E \oplus \mathcal{O}_{\mathbb{P}^{2}}(-4)\right)^{\vee} \otimes \mathcal{O}_{\mathbb{P}^{2}}(-4) \\
& =\left(\mathcal{O}_{\mathbb{P}^{2}} \oplus E^{\vee} \oplus \mathcal{O}_{\mathbb{P}^{2}}(4)\right) \otimes \mathcal{O}_{\mathbb{P}^{2}}(-4) \\
& =\mathcal{O}_{\mathbb{P}^{2}}(-4) \oplus E \oplus \mathcal{O}_{\mathbb{P}^{2}},
\end{aligned}
$$

$\mathcal{O}_{\mathbb{P}^{2}}(-4) \oplus E \oplus \mathcal{O}_{\mathbb{P}^{2}}$ admits a non zero global section, so $\omega_{X} \otimes \varphi^{*} \mathcal{O}_{\mathbb{P}^{2}}(-1)$ admits also non zero global section. Namely there is an effective divisor $Z$ such that $K_{X}=$ $\varphi^{*} H+Z$, where $H$ is the divisor of the hyperplane. Because

$$
p_{g}(X)=h^{2}\left(\varphi_{*} \mathcal{O}_{X}\right)=h^{2}\left(\mathcal{O}_{\mathbb{P}^{2}} \oplus E \oplus \mathcal{O}_{\mathbb{P}^{2}}(-4)\right)=h^{0}(E(1))+3=3 .
$$

$Z$ is the fixed part of $\left|K_{X}\right|$. It implies that

$$
K_{X}^{2} \geqslant\left(\varphi^{*} H\right)^{2}=36=9\left(p_{g}(X)+1\right) \geqslant 9 \chi\left(\mathcal{O}_{X}\right)
$$

by Miyaoka-Yau inequality, $K_{X}^{2}=36, Z=0$ and $q(X)=0$. So $\varphi_{K_{X}}=\varphi$. $\square$

LEMMA 3.2. If $\varphi=\varphi_{K_{X}}$ is a finite abelian cover of degree $d$ over $\mathbb{P}^{2}$, then $\varphi_{*} \mathcal{O}_{X}=\mathcal{O}_{\mathbb{P}^{2}} \oplus \mathcal{O}_{\mathbb{P}^{2}}(-2)^{\oplus d-2} \oplus \mathcal{O}_{\mathbb{P}^{2}}(-4)$.

Proof. Because $\varphi$ is an abelian cover, $\varphi_{*} \mathcal{O}_{X}$ is a direct sum of the line bundle.

$$
\varphi_{*} \mathcal{O}_{X}=\mathcal{O}_{\mathbb{P}^{2}} \oplus \bigoplus_{i=1}^{d-1} \mathcal{O}_{\mathbb{P}^{2}}\left(-l_{i}\right)
$$


Assume $0<l_{1} \leqslant l_{2} \leqslant \cdots \leqslant l_{d-1}$. Since $K_{X}=\varphi^{*}\left(\mathcal{O}_{\mathbb{P}^{2}}(1)\right)$, for any $m \geqslant 1$,

$p_{m}(X)=h^{0}\left(m K_{X}\right)=h^{0}\left(\varphi^{*}\left(\mathcal{O}_{\mathbb{P}^{2}}(m)\right)\right)=h^{0}\left(\mathcal{O}_{\mathbb{P}^{2}}(m)\right)+\sum_{i=1}^{d-1} h^{0}\left(\mathcal{O}_{\mathbb{P}^{2}}\left(m-l_{i}\right)\right)$.

Because $p_{g}(X)=p_{1}(X)=h^{0}\left(\varphi^{*}\left(\mathcal{O}_{\mathbb{P}^{2}}(1)\right)\right)=h^{0}\left(\mathcal{O}_{\mathbb{P}^{2}}(1)\right)=3$, we see that

$$
h^{0}\left(\mathcal{O}_{\mathbb{P}^{2}}\left(1-l_{i}\right)\right)=0, \forall i
$$

So $l_{i} \geqslant 2$.

And $h^{2}\left(\varphi_{*} \mathcal{O}_{X}\right)=h^{2}\left(\mathcal{O}_{\mathbb{P}^{2}}\right)+\sum_{i=1}^{d-1} h^{2}\left(\mathcal{O}_{\mathbb{P}^{2}}\left(-l_{i}\right)\right)$, so $3=\sum_{i=1}^{d-1} h^{0}\left(\mathcal{O}_{\mathbb{P}^{2}}\left(l_{i}-3\right)\right)$, then $l_{i} \leqslant 4$.

Then there are two cases:

(1) $l_{1}=\cdots=l_{d-2}=2, \quad l_{d-1}=4$, and

(2) $l_{1}=\cdots=l_{d-4}=2, \quad l_{d-3}=l_{d-2}=l_{d-1}=3$.

On the other hand, if $m=2$, we have the second plurigenus $p_{2}(X)=K_{X}^{2}+\chi\left(\mathcal{O}_{X}\right)=$ $d+4$. So

$$
d+4=h^{0}\left(\mathcal{O}_{\mathbb{P}^{2}}(2)\right)+\sum_{i=1}^{d-1} h^{0}\left(\mathcal{O}_{\mathbb{P}^{2}}\left(2-l_{i}\right)\right) .
$$

The second case does not satisfy the equation. So the lemma is proved.

Now suppose $\varphi: X \rightarrow \mathbb{P}^{2}$ be an abelian cover associated to an abelian group $G \cong \mathbb{Z}_{n_{1}} \oplus \cdots \oplus \mathbb{Z}_{n_{k}}$. Then $X$ is the normalization of the surface defined by

$$
z_{1}^{n_{1}}=f_{1}=\prod_{\alpha} p_{\alpha}^{\alpha_{1}}, \cdots, z_{k}^{n_{k}}=f_{k}=\prod_{\alpha} p_{\alpha}^{\alpha_{k}},
$$

where $p_{\alpha}$ 's are prime factors and $\alpha=\left(\alpha_{1}, \cdots, \alpha_{k}\right) \in G$. Denote $x_{\alpha}$ to be the degree of $p_{\alpha}, e_{i}=(0, \cdots, 0,1,0, \cdots, 0) \in G, 1 \leq i \leq k$, and $l_{g}$ be the degree of $L_{g}$. So $x_{\alpha}$ and $l_{g}$ are all integers. Then

$$
\begin{gathered}
n_{i} l_{e_{i}}=\sum_{\alpha} \alpha_{i} x_{\alpha} \quad i=1, \cdots k, \\
l_{g}=\sum_{i=1}^{k} g_{i} l_{e_{i}}-\sum_{\alpha}\left[\sum_{i=1}^{k} \frac{g_{i} \alpha_{i}}{n_{i}}\right] x_{\alpha} .
\end{gathered}
$$

Lemma 3.3. Using the notation as above, if $\varphi=\varphi_{K_{X}}$, then there exists $g^{\prime}=$ $\left(g_{1}^{\prime}, \cdots, g_{k}^{\prime}\right) \in G \cong \mathbb{Z}_{n_{1}} \oplus \cdots \oplus \mathbb{Z}_{n_{k}}$, such that $x_{\alpha}$ satisfies the following equations

$$
(*) \quad\left\{\begin{array}{l}
n_{i} l_{e_{i}}=\sum_{\alpha} \alpha_{i} x_{\alpha} \\
l_{g^{\prime}}=\sum_{i=1}^{k} g_{i}^{\prime} l_{e_{i}}-\sum_{\alpha}\left[\sum_{i=1}^{k} \frac{g_{i}^{\prime} \alpha_{i}}{n_{i}}\right] x_{\alpha}=4 \\
l_{g}=\sum_{i=1}^{k} g_{i} l_{e_{i}}-\sum_{\alpha}\left[\sum_{i=1}^{k} \frac{g_{i} \alpha_{i}}{n_{i}}\right] x_{\alpha}=2, \quad g \neq g^{\prime}, g \in G
\end{array}\right.
$$

Proof. It comes from Lemma 3.2 directly.

By the above lemma, finding surfaces whose canonical map are abelian covers over $\mathbb{P}^{2}$ is equivalent to finding the integral roots $\left\{x_{\alpha}\right\}$ of the above equations. 
THEOREM 3.4. If the canonical degree of $X$ is 36 , then $\varphi_{K_{X}}$ can not be an abelian cover.

Proof. From the famous theorem of Beauville([Bea]), we know the image of $\varphi_{K_{X}}(X)$ is $\mathbb{P}^{2}$. We assume that $\varphi_{K_{X}}: X \rightarrow \mathbb{P}^{2}$ is an abelian cover associated to abelian group $G$. Then $|G|=36$.

If $G=\mathbb{Z}_{36}$, then there exists some $g^{\prime} \in \mathbb{Z}_{36}$ and corresponding $l$ such that $\left\{x_{\alpha}\right\}$ must be satisfied the following equations:

$$
\left\{\begin{array}{l}
l_{g^{\prime}}=g^{\prime} l_{1}-\sum_{3=1}^{35}\left[\frac{g^{\prime} \alpha}{36}\right] x_{\alpha}=4, \\
l_{g}=g l_{1}-\sum_{\alpha=1}^{35}\left[\frac{g \alpha}{36}\right] x_{\alpha}=2, \quad g \neq g^{\prime}, \quad 1 \leq g \leq 35 \\
36 l_{1}=\sum_{\alpha=1}^{35} \alpha x_{\alpha} .
\end{array}\right.
$$

After computation, we find that there is no non-negative integral solution for the above equations for any $1 \leq g^{\prime} \leq 35$.

Using the same method, we find there is no non-negative integral solution for $(*)$ as $G=\mathbb{Z}_{n_{1}} \oplus \mathbb{Z}_{n_{2}}$ and $n_{1} \mid n_{2}$.

So $\varphi_{K_{X}}$ can not be abelian.

Theorem 3.5. Assume $\varphi: X \longrightarrow \mathbb{P}^{2}$ is an abelian cover.

(1) Let $\operatorname{deg} \varphi=16$, then $\varphi=\varphi_{K_{X}}$ if and only if $X$ is defined by

$$
z_{1}^{2}=\ell_{1} \ell_{3} \ell_{4} \ell_{7}, \quad z_{2}^{2}=\ell_{1} \ell_{2} \ell_{4} \ell_{5}, \quad z_{3}^{2}=\ell_{1} \ell_{2} \ell_{3} \ell_{6}, \quad z_{4}^{2}=\ell_{2} \ell_{5} \ell_{6} \ell_{8} .
$$

In particular, the surface defined by the first three equations is a Campedelli surface.

(2) Let $\operatorname{deg} \varphi=9$, then $\varphi=\varphi_{K_{X}}$ if and only if $X$ is defined by $z_{1}^{3}=a_{1} a_{2}^{2}, z_{2}^{3}=$ $a_{1} a_{2} a_{3}$.

(3) Let $\operatorname{deg} \varphi=8$, then $\varphi=\varphi_{K_{X}}$ if and only if $X$ is defined by either $z_{1}^{2}=$ $a_{1} a_{4}, z_{2}^{2}=a_{2} a_{4}, z_{3}^{2}=a_{3} a_{4}$, or $z_{1}^{2}=a_{1} a_{2}, z_{2}^{4}=a_{2}^{3} a_{3}$.

(4) Let $\operatorname{deg} \varphi=6$, then $\varphi=\varphi_{K_{X}}$ if and only if $X$ is defined by $z_{1}^{2}=a_{1} a_{2}, z_{2}^{3}=$ $a_{2} a_{3}^{2}$.

(5) Let $\operatorname{deg} \varphi=4$, then $\varphi=\varphi_{K_{X}}$ if and only if $X$ is defined by either $z^{4}=a_{1}^{2} b_{2}^{3}$,or $z_{1}^{2}=b_{1}, z_{2}^{2}=b_{2}$.

(6) Let $\operatorname{deg} \varphi=3$, then $\varphi=\varphi_{K_{X}}$ if and only if $X$ is defined by either $z^{3}=c_{1}^{2}$,or $z^{3}=c_{2}$.

(7) Let $\operatorname{deg} \varphi=2$, then $\varphi=\varphi_{K_{X}}$ if and only if $X$ is $z^{2}=h$.

Here $\ell_{i}$ 's define different lines and there are at most three lines among them passing through one point, for $i=1 \cdots 8 ; a_{i}$ 's are reduced of degree $2 ; b_{i}$ 's are reduced of degree $4 ; c_{1}, c_{2}$ are reduced of degree $6 ; h$ is reduced of degree 8 and all the irreducible components of $a_{i}$ 's,$b_{i}$ 's, $c_{1}, c_{2}$ and $h$ are simply normal crossing.

Proof. If $\varphi=\varphi_{K_{X}}$, then $K_{X}=\varphi^{*}\left(\mathcal{O}_{\mathbb{P}^{2}}(1)\right),\left|K_{X}\right|$ has no fixed part and is base point free.

Let $G=\mathbb{Z}_{n_{1}} \oplus \cdots \oplus \mathbb{Z}_{n_{k}}$. Since the arguments are similar, we only prove the most complicated case when $|G|=16$. By Lemma 3.3, we only need to find the integral solution of the equations $(*)$. After computation, we find if and only if $G=\mathbb{Z}_{2} \oplus \mathbb{Z}_{2} \oplus \mathbb{Z}_{2} \oplus \mathbb{Z}_{2}$, there are integral solutions.

(1) $l_{(1,0,0,0)}=4, x_{(1,1,0,1)}=x_{(1,1,1,0)}=x_{(1,0,1,1)}=x_{(1,0,1,0)}=x_{(1,0,0,1)}=$ $x_{(1,0,0,0)}=x_{(1,1,1,1)}=x_{(1,1,0,0)}=1, x_{(0,1,0,0)}=x_{(0,0,0,1)}=x_{(0,0,1,0)}=$ $x_{(0,0,1,1)}=x_{(0,1,1,0)}=x_{(0,1,0,1)}=x_{(0,1,1,1)}=0$, 
(2) $l_{(1,1,0,0)}=4, x_{(0,1,1,1)}=x_{(1,0,1,1)}=x_{(0,1,1,0)}=x_{(1,0,1,0)}=x_{(1,0,0,1)}=$ $x_{(0,1,0,0)}=x_{(1,0,0,0)}=x_{(0,1,0,1)}=1, x_{(0,0,1,1)}=x_{(1,1,0,0)}=x_{(1,1,1,1)}=$ $x_{(1,1,0,1)}=x_{(1,1,1,0)}=x_{(0,0,0,1)}=x_{(0,0,1,0)}=0$,

(3) $l_{(1,1,1,0)}=4, x_{(1,1,1,0)}=x_{(0,1,0,1)}=x_{(1,0,0,1)}=x_{(0,1,0,0)}=x_{(1,0,0,0)}=$ $x_{(1,1,1,1)}=x_{(0,0,1,0)}=x_{(0,0,1,1)}=1, x_{(0,1,0,1)}=x_{(1,1,0,0)}=x_{(0,1,1,0)}=$ $x_{(0,1,1,1)}=x_{(1,1,0,1)}=x_{(1,0,1,1)}=x_{(0,0,0,1)}=0$,

(4) $l_{(1,1,1,1)}=4, x_{(0,1,1,1)}=x_{(1,1,0,1)}=x_{(1,1,1,0)}=x_{(1,0,1,1)}=x_{(0,1,0,0)}=$ $x_{(1,0,0,0)}=x_{(0,0,1,0)}=x_{(0,0,0,1)}=1, x_{(0,0,1,1)}=x_{(1,0,1,0)}=x_{(1,1,0,0)}=$ $x_{(1,0,0,1)}=x_{(0,1,1,0)}=x_{(0,1,0,1)}=x_{(1,1,1,1)}=0$.

Although there are 4 sets of solution, these defining equations are equivalent after quotient the group $s l\left(4, \mathbb{Z}_{2}\right)$ which means that they are same surfaces. So the surface $X$ is defined by (3.3). Next we are going to discuss the configurations of $\ell_{i}$ 's as following three cases.

- If $\ell_{i}$ 's are in general position, i.e. they are simply normal crossing. Now we prove $X$ is smooth.

Let $p_{i j}$ be the intersection point of $\ell_{i}$ and $\ell_{j}$. The cover is locally defined by

$$
z_{1}^{2}=x^{a_{11}} y^{a_{12}}, \quad z_{2}^{2}=x^{a_{21}} y^{a_{22}}, \quad z_{3}^{2}=x^{a_{31}} y^{a_{32}}, \quad z_{4}^{2}=x^{a_{41}} y^{a_{42}},
$$

where $a_{i j}=0$ or 1 for all $i, j$.

It is easy to check that $\left\{\left(a_{i 1}, a_{i 2}\right)\right\} \nsubseteq\{(1,1),(0,0)\}$ i.e., at least one pair $\left\{\left(a_{i 1}, a_{i 2}\right)\right\}=\{(1,0)\}$ or $\{(0,1)\}$. Without lose of generality, we can assume $\left(a_{11}, a_{21}\right)=(1,0)$, i.e. $z_{1}^{2}=x$. The cover is branched along the smooth line. So the surface is smooth.

From Theorem 2.4, the ramification index of $H_{i}=\operatorname{Div}\left(\ell_{i}\right)$ is 2 . Thus $K_{X}=$ $\varphi^{*}\left(\mathcal{O}_{\mathbb{P}^{2}}(-3)+\frac{1}{2} \sum_{i=1}^{8} H_{i}\right)=\varphi^{*}\left(\mathcal{O}_{\mathbb{P}^{2}}(1)\right)$, which means $\varphi=\varphi_{K_{X}}$.

- If there are three of $\ell_{i}$ 's intersecting at a point, we blow up these triple points at first. Let $\sigma: P \rightarrow \mathbb{P}^{2}$ be the blowing-ups of these triple points with $\left\{E_{s}\right\}$ the except curves and $\pi: \Sigma \rightarrow P$ be the corresponding abelian cover, i.e., the pull back of $\varphi$ by $\sigma$. Then it is easy to check that $E_{s}$ 's are in the branch locus of $\pi$ and the ramification indices are also 2. Similarly, we can show that $\Sigma$ is smooth. Then the canonical divosor

$$
\begin{aligned}
K_{\Sigma} & =\pi^{*}\left(\sigma^{*}(-3 H)+\sum_{s} E_{s}+\frac{1}{2}\left(\sigma^{*}(8 H)-3 \sum_{s} E_{s}\right)+\frac{1}{2} \sum_{s} E_{s}\right) \\
& =\pi^{*} \sigma^{*}(H),
\end{aligned}
$$

where $H$ is a hyperplane on $\mathbb{P}^{2}$. So the surface is minimal and the degree of the canonical map is 16 . In this case, $X$ has only $A_{1}$ type singularities.

- If there are $d$ lines among $\ell_{i}$ 's passing through a point, $d \geq 4$. We blow up these points such that the branch locus are normal crossing. Let $\sigma: P \rightarrow$ $\mathbb{P}^{2}$ be the blowing-ups of these points with $\left\{E_{s} \mid s \in S\right\}$ the except curves and $\pi: \Sigma \rightarrow P$ be the corresponding abelian cover. Then $\Sigma$ is smooth. $\left\{E_{s} \mid s \in S\right\}$ decomposes into 2 parts: $\left\{E_{s} \mid s \in S_{1}\right\}$ are in the branch locus of 
$\pi$ and $\left\{E_{s} \mid s \in S_{2}\right\}$ are not. Then the canonical divisor

$$
\begin{aligned}
K_{\Sigma} & =\pi^{*}\left(\sigma^{*}(-3 H)+\sum_{s \in S} E_{s}+\frac{1}{2}\left(\sigma^{*}(8 H)-d \sum_{s \in S} E_{s}\right)+\frac{1}{2} \sum_{s \in S_{1}} E_{s}\right) \\
& =\pi^{*}\left(\sigma^{*}(H)+\frac{3-d}{2} \sum_{s \in S_{1}} E_{s}+\frac{2-d}{2} \sum_{s \in S_{2}} E_{s}\right),
\end{aligned}
$$

where $H$ is a hyperplane on $\mathbb{P}^{2}$. Obviously, $K_{\Sigma}^{2}<16$. There is no -1 curves on $\Sigma$ by resolution and the singularities on $X$ are not rational double points.

Therefore the configuration of $\ell_{i}$ has at most triple points.

Let $X_{1}$ be the surface defined by the first three equations of (3.3) and suppose $\pi^{\prime}: X_{1} \rightarrow \mathbb{P}^{2}$. When $\ell_{i}$ 's are in general position, we can show that $X_{1}$ is smooth and $K_{X_{1}}^{2}=2$. By Theorem 2.2, $L_{g} \sim 2 H$ for $g \neq 0$, where $H$ is a hyperplane on $\mathbb{P}^{2}$. So $h^{0}\left(-L_{g}\right)=h^{0}\left(K_{\mathbb{P}^{2}}+L_{g}\right)=0$ for all nonzero $g \in \mathbb{Z}_{2} \oplus \mathbb{Z}_{2} \oplus \mathbb{Z}_{2}$. Due to Corollary 2.3, $p_{g}\left(X_{1}\right)=q\left(X_{1}\right)=0$. Therefore the surface $X_{1}$ is a Campedelli surface.

Now we compute the fundamental group of the surface $X_{1}$.

According to the result of the canonical resolution of double cover, the preimage of each $L_{i}=\operatorname{Div}\left(\ell_{i}\right)$ by $\pi^{\prime}$ is irreducible. Then assume $\pi^{\prime *}\left(L_{i}\right)=2 A_{i}$, we can get $A_{i}^{2}=2$.

Due to $2 A_{i} \sim \pi^{\prime *}(H), 2\left(A_{i}-A_{j}\right) \sim 0$. Assume $A_{i} \sim A_{j}, i \neq j$, then $h^{0}\left(A_{i}\right) \leq 2$ since $h^{0}\left(A_{i}+A_{j}\right) \geq h^{0}\left(A_{i}\right)+h^{0}\left(A_{j}\right)-1$. And the equation holds if and only if $A_{i}$ and $A_{j}$ do not intersect, which is contradict to $A_{i}^{2}=2$. So $h^{0}\left(A_{i}\right)=1$, which means that $A_{i}$ is not linear equivalent to $A_{j}$.

Therefore each $A_{i}-A_{j}$ is a 2-torsion element in $X_{1}$. So $X_{1}$ has at least 62 -torsion elements.

Denote $B=\sum_{i=1}^{7} L_{i}$ to be the branch locus in $\mathbb{P}^{2} . \quad \pi_{1}\left(\mathbb{P}^{2}-B\right)=\mathbb{Z}^{\oplus}$ is an abelian group. We know that $X_{1}-\pi^{* *}(B)$ is an étale cover over $\mathbb{P}^{2}-B$. So $\pi_{1}\left(X_{1}-\pi^{\prime *}(B)\right)$ is a subgroup of $\pi_{1}\left(\mathbb{P}^{2}-B\right)$ which is abelian and $\pi_{1}\left(X_{1}\right)$ is the quotient group of $\pi_{1}\left(X_{1}-\pi^{*}(B)\right)$, then $\pi_{1}\left(X_{1}\right)$ is also an abelian group, which means $\pi_{1}\left(X_{1}\right)=H_{1}\left(X_{1}, \mathbb{Z}\right)$.

As $q=0, H^{1}\left(X_{1}, \mathbb{C}\right)=0$. Using the universal coefficient theorem for cohomology, we know $H^{1}\left(X_{1}, \mathbb{C}\right)=H_{1}\left(X_{1}, \mathbb{C}\right)=0$. Since $H_{1}\left(X_{1}, \mathbb{C}\right)=H^{1}\left(X_{1}, \mathbb{Z}\right) \otimes \mathbb{C}=0$, $H^{1}\left(X_{1}, \mathbb{Z}\right)$ only has torsion parts.

Due to the following exact sequence

$$
H^{1}\left(X_{1}, \mathcal{O}_{X_{1}}\right) \longrightarrow H^{1}\left(X_{1}, \mathcal{O}_{X_{1}}^{*}\right) \longrightarrow H^{2}\left(X_{1}, \mathbb{Z}\right) \longrightarrow H^{2}\left(X_{1}, \mathcal{O}_{X_{1}}\right)
$$

and $p_{g}\left(X_{1}\right)=q\left(X_{1}\right)=0$, we know $\operatorname{Pic} X_{1}=H^{1}\left(X_{1}, \mathcal{O}_{X_{1}}^{*}\right)=H^{2}\left(X_{1}, \mathbb{Z}\right)$.

From the following sequence

$$
0 \longrightarrow \operatorname{Ext}\left(H_{1}\left(X_{1}, \mathbb{Z}\right), \mathbb{Z}\right) \longrightarrow H^{2}\left(X_{1}, \mathbb{Z}\right) \longrightarrow \operatorname{Hom}\left(H_{2}\left(X_{1}, \mathbb{Z}\right), \mathbb{Z}\right) \longrightarrow 0
$$

we have $H_{1}\left(X_{1}, \mathbb{Z}\right)=\operatorname{Tor}\left(H^{2}\left(X_{1}, \mathbb{Z}\right)\right)$.

For any Campedelli surface $X$, it is well known that $\left|\pi_{1}(X)\right| \leqslant 9([$ Rei $])$. So the torsion group of the Picard group of $X_{1}$ is $G=\mathbb{Z}_{2} \oplus \mathbb{Z}_{2} \oplus \mathbb{Z}_{2}$, i.e., $\pi_{1}\left(X_{1}\right)=$ $\mathbb{Z}_{2} \oplus \mathbb{Z}_{2} \oplus \mathbb{Z}_{2}$.

\section{REMARK 3.6.}

(1) From the above theorem, we know the defining equations of Persson's surface.

(2) The Campedelli surface constructed in the proof is as same as that given by Kulikov ([Kul]). In fact, Campedelli surfaces with fundamental group of order 8 are completely classified in [ML-P-R]. 
COROLlary 3.7. If

$$
\varphi=\varphi_{K_{X}}: X \longrightarrow \mathbb{P}^{2}
$$

is an abelian cover, then $d$ is equal to $2,3,4,6,8,9$ or 16 .

4. Defining equations of Tan's examples. In [Tan], Tan constructed a series of nice surfaces whose canonical maps are of odd degrees by using a so-called conception $\mathbb{Z}_{p}$-set of the singularities.

Let $N$ be a set of singular points of surface $\Sigma$, and let $\sigma: Y \rightarrow \Sigma$ be the minimal resolution of $\Sigma$ with $\Gamma_{1}, \cdots, \Gamma_{n}$ the components of $\sigma^{-1}(N)$. If there are positive integers $a_{i}<p$ and a divisor $D$ on $Y$ such that $\sum_{i=1}^{n} a_{i} \Gamma \sim p D$ then $N$ is called a $\mathbb{Z}_{p}$-set of singularities on $\Sigma$. A $p$ fold cyclic cover can be determined by the $\mathbb{Z}_{p}$-set.

Take 3 points $q_{1}, q_{2}, q_{3}$ in general position on $\mathbb{P}^{2}$, and every 3 lines passing through each point. This configuration of 9 lines has $n+3$ triple points and $27-3 n$ double points. And he assumed that all lines passing through $q_{1}, q_{2}$ contain no 4 triple points. Blowing up the $n+3$ triple points, we suppose each $A_{i}$ to be the strict transforms of the 3 lines passing through $q_{i}$. Then a Galois triple cover $\pi_{1}$ with branch locus $A_{1}+A_{2}+A_{3}$ can be defined and $N=\pi_{1}^{-1}\left(\bigcup_{i<j} A_{i} \bigcap A_{j}\right)$ is a $\mathbb{Z}_{3}$-set. His example $X$ is the 3 fold cyclic cover determined by $N$. In order to show the canonical map of $X$ is of degree 3, Tan calculated the invariants of such surface. But the computation of the invariants of the surface is pretty complicated. The main part of [Tan] is the computation of $p_{g}(X)$.

We can reconstruct the Tan's example by explicitly equations. Let $\varphi: X \rightarrow \mathbb{P}^{2}$ be an abelian cover branched over the line configuration described by Tan. Assume $\ell_{1}, \ell_{2}, \ell_{3}$ are the 3 lines through $q_{1}, \ell_{4}, \ell_{5}, \ell_{6}$ are the 3 lines through $q_{2}$ and $\ell_{7}, \ell_{8}, \ell_{9}$ are the 3 lines through $q_{3} . \varphi$ is defined by the following equations.

$$
\pi: \quad z_{1}^{3}=\ell_{1} \ell_{2} \ell_{3} \ell_{4} \ell_{5} \ell_{6} \ell_{7} \ell_{8} \ell_{9}, \quad z_{2}^{3}=\ell_{1} \ell_{2} \ell_{3} \ell_{4}^{2} \ell_{5}^{2} \ell_{6}^{2} .
$$

Let $\sigma: P \rightarrow \mathbb{P}^{2}$ be blowing-ups of $q_{1}, q_{2}, q_{3} . \pi: X \rightarrow P$ is the pull back of $\varphi$ by $\sigma$. Let $\Sigma$ be the minimal resolution of $\pi^{\prime}: Y \rightarrow \mathbb{P}^{2}$ which is a cyclic cover defined by $z^{3}=\ell_{1} \ell_{2} \ell_{3} \ell_{4} \ell_{5} \ell_{6} \ell_{7} \ell_{8} \ell_{9}$. It is clear that $X$ and $Y$ have at most $A_{1}$-type singularities. We calculate invariants $q(\Sigma)=q(X)=0$ and $p_{g}(X)=p_{g}(\Sigma)=8-n$ by Theorem 2.2. The canonical map of $X$ factorizes through that of $\Sigma$, so it is of degree 3 .

In [Tan], the author also constructed a surface whose canonical map is of degree 5 by using $\mathbb{Z}_{5}$ set. We can also reconstruct it as follows.

Take five lines $\ell_{1}, \cdots, \ell_{5}$ in general position on $\mathbb{P}^{2} . \pi: X \rightarrow \mathbb{P}^{2}$ is an abelian cover defined by

$$
z_{1}^{5}=\ell_{1} \ell_{2} \ell_{3} \ell_{4} \ell_{5}, \quad z_{2}^{5}=\ell_{1} \ell_{2}^{2} \ell_{3}^{3} \ell_{4}^{4} .
$$

The 5 fold covering surface $\Sigma$ over $\mathbb{P}^{2}$ is defined by the first equation. Similarly, we get $K_{X}=25, K_{\Sigma}=5, \chi\left(\mathcal{O}_{X}\right)=\chi\left(\mathcal{O}_{\Sigma}\right)=5, p_{g}\left(\mathcal{O}_{X}\right)=p_{g}\left(\mathcal{O}_{\Sigma}\right)$. Therefore, the canonical map of $X$ factorizes through that of $\Sigma$. This means that the degree of the canonical map of $X$ is 5 .

\section{REFERENCES}

[Bea] A. Beauville, L'application canonique pour les surfaces de type général, Invention Math., 55 (1979), pp. 121-140. 
[Cat1] F. CATANESE, Babbage's conjecture, contact of surfaces, symmetric determinatal varieties and applications, Invent. Math., 63 (1981), pp. 433-465.

[Cat2] F. Catanese, On the moduli spaces of surfaces of general type, J. Differential Geom., 19 (1984), pp. 483-515.

[Com] A. Comessatti, Sulle superfici multiple cicliche, Rend. Sem. Mat. Univ. Padova, 1 (1930), pp. 1-45

[Gao] Y. GAO, A note on finite abelian cover, Science in China, Series A: Mathematics, 54 (2011), pp. 1333-1342.

[Kul] VIK. S. KULIKOV, Old examples and a new example of surfaces of general type with $p_{g}=$ 0, (Russian) Izv. Ross. Akad. Nauk Ser. Mat., 68 (2004), pp. 123-170; translation in Izv. Math., 68 (2004), pp. 965-1008.

[ML-P-R] M. Mendes Lopes, R. PARdini, And M. Reid, Campedelli surfaces with fundamental group of order 8, Geom. Dedicata, 139 (2009), pp. 49-55.

[Par1] R. PARdini, Canonical images of surfaces, J. reine angew. Math., 417 (1991), pp. 215219.

[Par2] R. PARdini, Abelian covers of algebraic varieties, J. Reine Angew. Math., 417 (1991), pp. 191-213.

[Per] U. Persson, Double coverings and surfaces of general type., In: Olson, L.D.(ed.) Algebraic geometry, Lect. Notes Math., vol. 732, pp. 168-175, Berlin Heidelberg New York: Springer 1978

[Pet] C. A. M. Peters, On two types of surfaces of general type with vanishing geometric genus, Inventiones Math., 417 (1991), pp. 191-213.

[Rei] M. REID, Surfaces with $p_{g}=0, K^{2}=2$, preprint available at http://www.warwick.ac. $\mathrm{uk} / \sim \operatorname{masda} / \mathrm{surf} /$.

[Tan] S.-L. TAN, Surfaces whose canonical maps are of odd degrees, Math. Ann., 292 (1992), pp. 13-29.

[V-Z] G. VAN Der Geer And D. Zagier, The Hilbert modular group for the field $\mathbb{Q}[\sqrt{13}]$, Invent. Math., 42 (1977), pp. 93-134.

[Xiao] G. XIAO, Algebraic surfaces with high canonical degree, Math. Ann., 274 (1986), pp. 473483. 